\title{
The Infiltration of Reasonable Reasoning in Primary School Mathematics
}

\author{
Kailai Jiang, Yiran Zhang \\ Zhejiang Normal University, Jinhua, China \\ Email:3206200635@qq.com
}

How to cite this paper: Jiang, K.L. and Zhang, Y.R. (2021) The Infiltration of Reasonable Reasoning in Primary School Mathematics. Open Access Library Journal, 8: e7531.

https://doi.org/10.4236/oalib.1107531

Received: May 17, 2021

Accepted: June 13, 2021

Published: June 16, 2021

Copyright $\odot 2021$ by author(s) and Open Access Library Inc.

This work is licensed under the Creative

Commons Attribution International

License (CC BY 4.0).

http://creativecommons.org/licenses/by/4.0/

(c) (i) Open Access

\begin{abstract}
This paper focused on the infiltration of reasonable reasoning in primary school mathematics. It firstly introduced the definition of rational reasoning, then discussed the presentation of reasonable reasoning in the primary school mathematics teaching material with three examples, and finally analyzed the thinking characteristics of a primary school student and put forward three suggestions for teachers.
\end{abstract}

\section{Subject Areas}

Mathematics Education

\section{Keywords}

Reasonable Reasoning, Mathematical thinking, Primary School Mathematics

\section{Introduction}

Reasoning as a priority in the primary school mathematics teaching is paid great attention by teachers, and students thinking development level and the requirement of mathematics learning "re-creation" determines the acquisition of knowledge should be given priority to with reasoning, but why put so high status of the ratiocination, reasoning the mathematical thought to the primary student's thinking training and what role? This study will integrate and analyze the previous studies and conclude the effect of reasonable reasoning on the cultivation of students' thinking.

\section{The Definition of Rational Reasoning}

Reasonable reasoning is the reasoning process of inferring certain results based on the existing facts and correct conclusions (including definitions, axioms, 
theorems, etc.), the results of experiments and practices, as well as personal experience and intuition. Conjecture is the most common and important way of thinking of reasonable reasoning. Induction and analogy first contain the element of conjecture. As a method of mathematical discovery, this way of thinking is non-logical deduction and a reasonable and plausible reasoning process, namely reasonable reasoning [1].

There is a variety of reasonable reasoning methods in mathematics, among which analogical reasoning and inductive reasoning are the most commonly used.

\subsection{Analogical Reasoning}

When explaining this way of thinking, Bolia emphasized two points: First, analogy is a kind of similarity, which is also a kind of fuzzy consistency; second is to translate the similarities into clear and definite concepts.

Analogical reasoning is also divided into three kinds, namely from the individual to the general generalization, some characteristics of the analogical reasoning and methodological analogical reasoning, these methods play a very important role in mathematics learning.

\subsection{Inductive Reasoning}

Inductive reasoning in reasonable reasoning refers to a thinking method from individual to general, from empirical fact or experimental fact to theory, which is also called empirical induction. In primary and secondary education, the function of induction is divided into two kinds, that is, the conclusion of finding problems and the path of solving mathematical problems.

\section{Reasonable Reasoning in the Concrete Presentation of Primary School Mathematics Textbooks}

\subsection{The Presentation of Reasonable Reasoning in "Numbers and Algebra"}

As an important part of the primary school mathematics curriculum system, "Mathematics and Algebra" has very obvious basic characteristics. In primary school mathematics teaching, the use of reasonable reasoning way, not only can realize the guidance of students to study mathematics, cultivate students' interest in mathematics learning, but also can make students' innovation consciousness has been continuously improved [2]. Through the analysis of the content of this part of the primary school mathematics textbook of the People's Education Edition, the author found that its general content mainly involves the knowledge points of integer, decimal, fraction, integer addition, subtraction, multiplication and division, negative number $\mathrm{D}$ and so on.

\section{Example 1:}

In the first section of "Re-understanding of Fractions" in the first volume of the fifth grade edition of Beijing Normal University, reasonable and reasoning thinking mode is applied in the three links of the textbook design. In the first 
step, the textbook uses three figures to represent three quarters and then guides the students to discover.

More than one graph can be used to represent fractions, which leads to the concept of integers; the second step is to observe that different graphs can be used to represent the same score, so as to deduce that the whole represented by the same score may be different. The third step is to use the situation of "holding a pen" to deduce that for different groups, the same score may represent different results.

In the above teaching process, students become observers, analysts, and comparators. After getting familiar with the relationship between numbers and figures, they can find the corresponding laws and get the relationship between the whole and the score. Reasonable reasoning can be used in "number understanding", "number operation", "proportion and equation" and so on, so as to ensure that students can learn mathematics courses in a better way, better use of reasonable reasoning thinking mode to get the required results, and cultivate students' mathematical thinking.

\subsection{The Presentation of Reasonable Reasoning in "Graphics and Geometry"}

"Graphics and Geometry" uses sensible reasoning in many ways. The "figure and geometry" in the primary school curriculum mainly involves the knowledge of the figure, the measure of the figure, the movement of the figure, and the position of the figure. The main purpose of this kind of course content is to guide students to have a better understanding of plane graphics, three-dimensional graphics and the relationship between them, on the basis of reasonable reasoning, to realize the necessary knowledge learning, master the necessary skills, learn to connect knowledge, and realize the establishment of a spatial concept.

\section{Example 2:}

In the second volume of the fifth grade edition of Beijing Normal University, every link of the textbook uses a reasonable and reasoning way of thinking. First, students can understand the space concept of cubes by folding and expanding the cube model.

Read, and then give the folded plane graphics, let the students use reasonable reasoning way of thinking, to derive the cube each.

What the surface looks like, or derive the geometric shape of a non-cube.

In the above learning activities, students, as the main body of the activities, under the guidance of the teacher, used the folding method by themselves to gradually explore the relationship between each face after folding, and reasonable reasoning thought permeated in the process.

\subsection{The Presentation of Reasonable Reasoning in "Statistics and Probability"}

"Statistics and probability" is an important content of primary school mathematics curriculum system. This part focuses on the possibility of the occurrence 
of the event after data collection, description and analysis, so as to make a more correct decision [3]. In the whole process of data collection and reasoning of students, reasonable reasoning mathematical thought has been used all the time, and teachers should also consciously infiltrate reasonable reasoning mathematical thought into teaching.

\section{Example 3:}

Taking the first lesson "Data Represent and Analysis", Unit 8, Volume 2, Grade 5, Beijing Normal University edition as an example, the teaching objective is to guide students to have a preliminary understanding of the relevant knowledge of statistics so that students know how to interpret information by using charts, and can correctly analyze the relevant information of charts, so as to draw relevant conclusions. In the four links of textbook design, each link is composed of three parts: obtaining data, analyzing data and drawing conclusions. In the process of learning, I also have a certain understanding of the bar statistics chart. Reasonable thinking has always played a key role in the process of data analysis. For example, by analyzing air quality data from June to September in different years, middle school students can infer which year has the best air quality.

Through the analysis of examples, it can be concluded that the knowledge of "statistics and probability" is characterized by strong logic and tends to conduct statistical analysis of an object. It needs to have a comprehensive and deeper cognition of the event from the perspective of overall planning, and then the results can be presented in a more specific way.

\section{How to Infiltrate Reasonable Reasoning Thinking Mode in Teaching}

\subsection{The Thinking Characteristics of Primary School Students}

Some scholars point out that the mathematical thinking of pupils has image, flexibility and situation [3]. These several characteristics, the author combined with the analysis of scholars to make the following explanation.

\subsubsection{Visualization of Thinking}

In the six years of primary school, the level of concrete image thinking and abstract logic thinking of primary school students has been developing. For students of different ages, the development of their mathematical image thinking ability is very different. These thinking characteristics affect students' understanding degree of mathematical knowledge and their level of abstract thinking. Students can also often use thinking methods such as comparison, analysis, synthesis, abstraction, generalization, judgment and reasoning. They have basic logical mathematics and image-thinking ability, which is an indispensable part of mathematical thinking ability.

\subsubsection{Flexibility of Mind}

Students in mathematics learning to use before mathematical knowledge to explain the new knowledge, so as to deepen the understanding of new knowledge, 
this characteristic of thinking actually contains the analogical reasoning of reasoning thinking, flexibility is not strong, but if the students thinking will be plunged into mind-set, difficult to understand new knowledge, So teachers should pay attention to cultivating students' flexibility of thinking. And students in the process of mathematical analysis and reasoning, will analyze and adjust their reasoning process, find their own mistakes and correct, this kind of thinking characteristics of reasonable reasoning thinking has a very important role in the training, teachers need to improve the flexibility of students mathematical thinking training.

\subsubsection{Situational Thinking}

In the students' mathematics learning, if the situation and the learning materials that attract students are created, the students' interest in learning will be greatly enhanced, and the enthusiasm and initiative of students' thinking activities will be greatly enhanced, which will promote the cultivation and development of students' mathematical thinking and stimulate the activity of students' mathematical thinking.

\subsection{Enlightenments for Teachers in Class}

\subsubsection{Should Be Good at Choosing Inductive or Analogy Way to Train Students' Logical Reasoning Accomplishment [4]}

When teachers are teaching mathematics, when mathematical knowledge is abstracted from real objects or direct relations, they can use the inductive way to teach, and in the teaching process, they can permeate the idea of inductive reasoning to the students, and they can give the main body of the class to the students, and the teacher will play a guiding role. When a kind of knowledge is not completely unfamiliar to students, but can be extended from the existing objects or old knowledge, the method of analogy can be used for teaching, so that students can get the infiltration of the thinking mode of analogy reasoning in the process of learning.

\subsubsection{Replace "Acceptance" with "Reasoning"}

There are many principles and theorems in the study of mathematics. In the course of class, teachers often use the way of "telling" to teach that is, directly tell students the knowledge, but such students' learning is often in a passive "receiving" state, which is unfavorable for students to master the knowledge. In order to cultivate the students' reasoning ability, we can in class the inference process of theorem to students, teachers need only a small part of the theorem to the students, to guide the students' reasoning process, students can through their own efforts to understand the knowledge, not only can deepen students' understanding of the knowledge, can also be reasonable reasoning ability of this way of thinking into the teaching.

\subsubsection{From "Reasonable" to "Logical"}

In mathematics teaching, logical reasoning and deductive reasoning have dif- 
ferent functions. Reasonable reasoning is mainly used to explore ideas and discover conclusions; Deductive reasoning is mainly used to test conjectures and prove conclusions. They are interrelated and complement each other in the "conjecture-demonstration" of the achievement of new knowledge exploration [5].

Therefore, the infiltration of reasoning ability in primary school mathematics should be based on reasonable reasoning, focusing on cultivating students' reasonable reasoning ability and infiltrating the thinking methods of deductive reasoning. Only in this way can students realize the importance of both abilities.

\section{Conclusion}

Reasonable reasoning plays a very important role in primary school mathematics. How reasonable reasoning penetrates into primary school mathematics is explored by the way of text research. Firstly, the definition of the ratiocination of reasoning is classified. Then reasonable reasoning in the concrete presentation of primary school mathematics textbooks with three examples is discussed. Finally, to answer how to infiltrate reasonable reasoning thinking mode in teaching, we analyzed the thinking characteristics of a primary school student and put forward the following suggestions for teachers: Choosing inductive or analogy way to train students' logical reasoning accomplishment, replacing "acceptance" with "reasoning" and changing the thinking mode from "reasonable" to "logical".

\section{Conflicts of Interest}

The authors declare no conflicts of interest regarding the publication of this paper.

\section{References}

[1] Four Clean-Ups, Shipment. (2012) "Reasoning" Analysis. Journal of Curriculum, Teaching Materials, Teaching Methods, 32, 54-57.

[2] Wang, Q.G. (2016) Representation of Reasonable Reasoning in Primary School Mathematics Textbook. Teaching and Management, 32, 52-54.

[3] Liu, Y.M. (2007) Research on the Characteristics of Pupils' Mathematical Thinking. Theories, 128-129.

[4] Wu, W.W. and Shao, G.H. (2019) How to Implement Core Literacy of Logical Reasoning in Primary School Mathematics Classroom. Curriculum Teaching Materials, 39, 88-95.

[5] Zhou, L.D. (2016) Reasoning in Primary School Mathematics and Its Teaching. Shanghai Educational Research, 90-92+61. 